\title{
Weak convergence of the supremum distance for supersmooth kernel deconvolution
}

\author{
Bert van Es \\ Korteweg-de Vries Institute for Mathematics \\ Universiteit van Amsterdam \\ Plantage Muidergracht 24 \\ 1018 TV Amsterdam \\ The Netherlands \\ vanes@science.uva.nl \\ Shota Gugushvili \\ Eurandom \\ Technische Universiteit Eindhoven \\ P.O. Box 513 \\ 5600 MB Eindhoven \\ The Netherlands \\ gugushvili@eurandom.tue.nl
}

November 9, 2018

\begin{abstract}
We derive the asymptotic distribution of the supremum distance of the deconvolution kernel density estimator to its expectation for certain supersmooth deconvolution problems. It turns out that the asymptotics are essentially different from the corresponding results for ordinary smooth deconvolution.
\end{abstract}

Keywords: Deconvolution, kernel density estimator, Rayleigh distribution, supremum distance.

AMS subject classification: $62 \mathrm{G} 07$ 


\section{Introduction and results}

Consider the classical deconvolution problem: let $X_{1}, \ldots, X_{n}$ be i.i.d. observations, where $X_{i}=Y_{i}+Z_{i}$ and $Y_{i}$ and $Z_{i}$ are independent. Assume that the unobservable $Y_{i}$ have distribution function $F$ and density $f$, and that the random variables $Z_{i}$ have a known density $k$. Note that the density $g$ of $X_{i}$ is equal to the convolution of $f$ and $k$. The nonparametric deconvolution problem is the problem of estimating $f$ or $F$ from the observations $X_{i}$. Thus we want to recover the distribution of $Y_{i}$ using the contaminated measurements $X_{i}$. Additional information on measurement error models and many practical examples can be found in Carroll et al. (2006).

A popular density estimator for this problem is the deconvolution kernel density estimator introduced in Carroll and Hall (1988) and Stefanski and Carroll (1990). This estimator is defined as

$$
f_{n h}(x)=\frac{1}{2 \pi} \int_{-\infty}^{\infty} e^{-i t x} \frac{\phi_{w}(h t) \phi_{e m p}(t)}{\phi_{k}(t)} d t=\frac{1}{n h} \sum_{j=1}^{n} v_{h}\left(\frac{x-X_{j}}{h}\right),
$$

with

$$
v_{h}(u)=\frac{1}{2 \pi} \int_{-\infty}^{\infty} \frac{\phi_{w}(s)}{\phi_{k}(s / h)} e^{-i s u} d s .
$$

Here $w$ denotes a kernel function, $h>0$ is a bandwidth, $\phi_{\text {emp }}$ is the empirical characteristic function of the sample defined by $\phi_{e m p}(t)=(1 / n) \sum_{j=1}^{n} e^{i t X_{j}}$, and $\phi_{w}$ and $\phi_{k}$ denote the characteristic functions of $w$ and $k$, respectively. Note that (11) is not a standard kernel density estimator, because the kernel function $v_{h}$ depends on the bandwidth $h$. For an introduction to the estimator (11) see e.g. Wand and Jones (1995).

The rate of decay to zero at minus and plus infinity of the modulus of the characteristic function $\phi_{k}$, and consequently the smoothness of $k$, is crucial to the asymptotic behaviour of (11). Two cases have been distinguished, the ordinary smooth case, where $\left|\phi_{k}\right|$ decays algebraically to zero, and the supersmooth case, where it decreases exponentially. The asymptotics in the ordinary smooth case are essentially the same as for a kernel estimator of a higher order derivative of a density, see e.g. Fan (1991), Fan and Liu (1997) and van Es and Kok (1998). The asymptotics in the supersmooth case have been studied e.g. in Fan (1991) and van Es and Uh (2004, 2005).

Notice that the above papers study local properties of the estimator (1), i.e. its pointwise behaviour. We, on the other hand, will focus on the asymptotic behaviour of the supremum distance of the estimator to its expectation, which provides a global measure of its performance. Accordingly, define

$$
M_{n}=\sup _{0 \leq x \leq 1}\left|f_{n h}(x)-\mathrm{E}\left[f_{n h}(x)\right]\right| .
$$

The fact that the supermum is taken over $[0,1]$ is not a restriction of generality and is for convenience only. One could have considered any interval 
$[a, b]$. An alternative here is to consider the integrated squared error of the estimator $f_{n h}$. This was done in Holzmann and Boysen (2006).

The asymptotic distribution of the supremum distance similar to (2), namely $\sup _{x \in[0,1]}(g(x))^{-1 / 2}\left|g_{n h}(x)-\mathrm{E}\left[g_{n h}(x)\right]\right|$, for an ordinary kernel density estimator $g_{n h}$ in the direct density estimation setting (i.e. in the errorfree case) was derived in Bickel and Rosenblatt (1973). Owing in a certain sense to the similarity of the asymptotics in the ordinary smooth deconvolution problem to that in the direct density estimation problem, qualitatively similar results were obtained in Bissantz et al. (2007) in the ordinary smooth deconvolution problem for the supremum distance $\sup _{x \in[0,1]}(g(x))^{-1 / 2} \mid f_{n h}(x)-$ $\mathrm{E}\left[f_{n h}(x)\right] \mid$. Normalisation with $\sqrt{g(x)}$ is explainable by the fact that the expression for the asymptotic variance in the asymptotic normality theorem for the estimator $f_{n h}(x)$ in the ordinary smooth deconvolution problem involves $g(x)$, see Fan (1991). No direct extension of the methods used in Bickel and Rosenblatt (1973) to the supersmooth deconvolution problem is possible and derivation of the asymptotic distribution of (2) requires a different approach. This is precisely the task of the present paper. Notice that in (2) we do not have to normalise with $\sqrt{g(x)}$, because the asymptotic variance in the asymptotic normality theorem for this case does not depend on $g$, but only on the error density $k$ (in some global way), see van Es and Uh $(2005)$.

We now state the conditions on the density $k$ and kernel $w$, which will be used throughout the paper. The condition on $k$ which defines supersmooth deconvolution is given in Condition 1 .

Condition 1. Assume that

$$
\phi_{k}(t)=C|t|^{\lambda_{0}} \exp \left[-|t|^{\lambda} / \mu\right]\left(1+o\left(|t|^{-1}\right)\right)
$$

as $|t| \rightarrow \infty$, for a constant $0<\lambda \leq 2$ and some constants $\mu>0, \lambda_{0} \in \mathbb{R}$ and $C \in \mathbb{R}$. Furthermore, let $\phi_{k}(t) \neq 0$ for all $t \in \mathbb{R}$.

Condition 11 is stronger than the usual condition on $k$ in supersmooth deconvolution given e.g. in van Es and Uh (2005), where the term $o\left(|t|^{-1}\right)$ is not present and one just has the asymptotic equivalence.

Condition 2. Let $\phi_{w}$ be real-valued, symmetric and have support $[-1,1]$. Let $\phi_{w}(0)=1$, and assume $\phi_{w}(1-t)=A t^{\alpha}+o\left(t^{\alpha}\right)$ as $t \downarrow 0$ for some constants $A$ and $\alpha \geq 0$.

For examples of such kernels see for instance van Es and Uh (2005).

The next theorem establishes the asymptotic distribution of $M_{n}$, which could prove useful for the construction of uniform confidence bands around $f$. Since it will appear repeatedly in the paper, we will write $\zeta(h)$ for $\exp \left(1 /\left(\mu h^{\lambda}\right)\right)$. 
Theorem 1. Assume Condition 1 for $\lambda=2$ and Condition 2 and let $\mathrm{E}\left[X_{j}^{2}\right]<\infty$. Let $V$ denote a positive random variable with a Rayleigh distribution with density $f_{V}(x)=x \exp \left[-x^{2} / 2\right] I_{[x \geq 0]}$. Then, as $n \rightarrow \infty$ and $h \rightarrow 0$,

$$
\frac{\sqrt{n}}{h^{\lambda(1+\alpha)+\lambda_{0}-1} \zeta(h)} M_{n} \stackrel{\mathcal{D}}{\rightarrow} \frac{1}{2} \sqrt{2} \frac{A}{\pi C}\left(\frac{\mu}{\lambda}\right)^{1+\alpha} \Gamma(\alpha+1) V
$$

where $\Gamma$ denotes the gamma function.

By assuming $\lambda=2$ we restrict ourselves to deconvolution problems for error distributions with characteristic functions that have an exponential tail like the characteristic function of a normal density. The most important case covered by this condition is standard normal deconvolution, where $\lambda=$ $2, \lambda_{0}=0, \mu=2$ and $C=1$. The condition $\lambda=2$ seems to be essential in the proof of Lemma 3, specifically in (8), where we prove a condition for tightness of the remainder process $R_{n}^{(1)}$. Whether it can be relaxed by other approaches, avoiding tightness, remains open.

The rate of convergence in Theorem 1 once again reflects the difficulty of the supersmooth deconvolution problem compared to the ordinary smooth deconvolution. Furthermore, unlike in ordinary smooth deconvolution, see Bissantz et al. (2007), in order to obtain the asymptotic distribution of $M_{n}$, we do not have to subtract a drift term. This also has a parallel when considering the asymptotics of the ISE $\left[f_{n h}\right]$ in the supersmooth deconvolution, see Holzmann and Boysen (2006) for additional details. Notice also that unlike the direct density estimation or the ordinary smooth deconvolution, see Bickel and Rosenblatt (1973) and Bissantz et al. (2007), the limit distribution in (4) is not Gumbel, which confirms the conjecture in Bissantz et al. (2007) for the case $\lambda=2$.

\section{Proof of Theorem 1}

The proof of Theorem 1 is based on a decomposition of $f_{n h}(x)$ in van Es and Uh (2005), which is the basis of the proof of their asymptotic normality theorem. We have

$$
\begin{aligned}
f_{n h}(x) & =\frac{1}{\pi C} h^{\lambda_{0}-1} \int_{\epsilon}^{1} \phi_{w}(s) s^{-\lambda_{0}} \exp \left(s^{\lambda} /\left(\mu h^{\lambda}\right)\right) d s \frac{1}{n} \sum_{j=1}^{n} \cos \left(\frac{X_{j}-x}{h}\right) \\
& +R_{n}^{(1)}(x)+R_{n}^{(2)}(x)+R_{n}^{(3)}(x),
\end{aligned}
$$


where $R_{n}^{(l)}(x)=(1 / n) \sum_{j=1}^{n} R_{n, j}^{(1)}(x), l=1,2,3$, and

$$
\begin{aligned}
R_{n, j}^{(1)}(x)= & \frac{1}{C} \frac{1}{\pi} h^{\lambda_{0}-1} \int_{\epsilon}^{1}\left(\cos \left(s\left(\frac{X_{j}-x}{h}\right)\right)-\cos \left(\frac{X_{j}-x}{h}\right)\right) \\
& \times \phi_{w}(s) s^{-\lambda_{0}} \exp \left(s^{\lambda} /\left(\mu h^{\lambda}\right)\right) d s \\
R_{n, j}^{(2)}(x)= & \frac{1}{2 \pi h} \int_{-\epsilon}^{\epsilon} \exp \left(i s\left(\frac{X_{j}-x}{h}\right)\right) \phi_{w}(s) \frac{1}{\phi_{k}(s / h)} d s \\
R_{n, j}^{(3)}(x)= & \frac{1}{2 \pi h}\left(\int_{-1}^{-\epsilon}+\int_{\epsilon}^{1}\right) \exp \left(i s\left(\frac{X_{j}-x}{h}\right)\right) \phi_{w}(s) \\
& \times\left(\frac{1}{\phi_{k}(s / h)}-\frac{1}{C}\left(\frac{|s|}{h}\right)^{-\lambda_{0}} \exp \left(|s|^{\lambda} /\left(\mu h^{\lambda}\right)\right)\right) d s .
\end{aligned}
$$

We will write $R_{n}^{(l)}, l=1,2,3$ for the stochastic processes $R_{n}^{(l)}=\left(R_{n}^{(l)}(x)\right)_{x \in[0,1]}$. Notice that these processes belong to the space $C[0,1]$.

Now the rough idea is to derive the asymptotic distribution of the supremum of the first summand in (5) minus its expectation and to show that the remainder terms are negligible. Define the process $U_{n}$ as $U_{n}(x)=$ $n^{-1 / 2} \sum_{j=1}^{n} U_{n, j}(x)$, where $U_{n, j}(x)=\cos \left(\left(X_{j}-x\right) / h\right)-\mathrm{E}\left[\cos \left(\left(X_{j}-x\right) / h\right)\right]$. Note that this is a process with expectation equal to zero at every $x$. Write

$$
S_{n}=\sup _{0 \leq x \leq 1}\left|U_{n}(x)\right| .
$$

Lemma 1. Under the conditions of Theorem 1 we have, as $n \rightarrow \infty$ and $h \rightarrow 0$,

$$
S_{n} \stackrel{\mathcal{D}}{\rightarrow} \sup _{0 \leq x \leq 2 \pi}|W(x)|,
$$

where $W$ is a zero mean Gaussian process on $[0,2 \pi]$ with covariance function $\operatorname{Cov}\left(W\left(x_{1}\right), W\left(x_{2}\right)\right)=(1 / 2) \cos \left(x_{1}-x_{2}\right)$.

Proof. Replacing $x$ by $y h$, by the periodicity of the cosine function we have 
for $h \leq(2 \pi)^{-1}$ that

$$
\begin{aligned}
S_{n} & =\sup _{0 \leq x \leq 1}\left|U_{n}(x)\right| \\
& =\sup _{0 \leq x \leq 1}\left|\frac{1}{\sqrt{n}} \sum_{j=1}^{n}\left(\cos \left(\frac{X_{j}-x}{h}\right)-\mathrm{E}\left[\cos \left(\frac{X_{j}-x}{h}\right)\right]\right)\right| \\
& =\sup _{0 \leq y \leq 1 / h}\left|\frac{1}{\sqrt{n}} \sum_{j=1}^{n}\left(\cos \left(\frac{X_{j}-y h}{h}\right)-\mathrm{E}\left[\cos \left(\frac{X_{j}-y h}{h}\right)\right]\right)\right| \\
& =\sup _{0 \leq y \leq 1 / h}\left|\frac{1}{\sqrt{n}} \sum_{j=1}^{n}\left(\cos \left(Y_{j}-y\right)-\mathrm{E}\left[\cos \left(Y_{j}-y\right)\right]\right)\right| \\
& =\sup _{0 \leq y \leq 2 \pi}\left|\frac{1}{\sqrt{n}} \sum_{j=1}^{n}\left(\cos \left(Y_{j}-y\right)-\mathrm{E}\left[\cos \left(Y_{j}-y\right)\right]\right)\right|, \\
& =\sup _{0 \leq y \leq 2 \pi}\left|W_{n}(y)\right|,
\end{aligned}
$$

where $Y_{j}=\left(X_{j} / h\right) \bmod 2 \pi$ and the process $W_{n}$ on $[0,2 \pi]$ is given by $W_{n}(y)=$ $n^{-1 / 2} \sum_{j=1}^{n}\left(W_{n, j}(y)-\mathrm{E}\left[W_{n, j}(y)\right]\right)$ with $W_{n, j}(y)=\cos \left(Y_{j}-y\right)$.

By Lemma 6 of van Es and Uh (2005) we know that $Y_{j} \stackrel{\mathcal{D}}{\rightarrow} \operatorname{Un}(0,2 \pi)$ as $h \rightarrow 0$ for each $j$, where $\operatorname{Un}(0,2 \pi)$ denotes the uniform distribution on $[0,2 \pi]$. Hence by the dominated convergence theorem we get that

$$
\begin{aligned}
& \operatorname{Cov}\left(\cos \left(Y_{j}-y_{1}\right), \cos \left(Y_{j}-y_{2}\right)\right) \\
& \rightarrow \frac{1}{2 \pi} \int_{0}^{2 \pi} \cos \left(u-y_{1}\right) \cos \left(u-y_{2}\right) d u=\frac{1}{2} \cos \left(y_{1}-y_{2}\right) .
\end{aligned}
$$

It follows that we have to study the convergence of the process $W_{n}(x)-$ $\mathrm{E}\left[W_{n}(x)\right]$ which belongs to $C[0,2 \pi]$. According to Prohorov's theorem and in particular Theorem 8.1 of Billingsley (1968), it suffices to show weak convergence of the finite dimensional distributions and tightness of the sequence. By the multivariate central limit theorem in the triangular array scheme or Cramer-Wold device, see Theorem 7.7 in Billingsley (1968), the finite dimensional distributions of the process $W_{n}$ converge to multivariate normal distributions with covariances given by $\operatorname{Cov}\left(W\left(y_{1}\right), W\left(y_{2}\right)\right)=(1 / 2) \cos \left(y_{1}-y_{2}\right)$. To prove tightness, we will verify conditions of Theorem 12.3 of Billingsley (1968). First of all, notice that the sequence $W_{n}(0)$ is tight, because the asymptotic normality of $W_{n}(0)$ follows by a univariate Lyapunov central limit theorem in a trinagular array scheme, see Theorem 7.3 in Billingsley 
(1968). Furthermore, for an arbitrary positive $\eta$,

$$
\begin{aligned}
& P\left(\left|W_{n}\left(y_{2}\right)-\mathrm{E}\left[W_{n}\left(y_{2}\right)\right]-\left(W_{n}\left(y_{1}\right)-\mathrm{E}\left[W_{n}\left(y_{1}\right)\right]\right)\right| \geq \eta\right) \\
& \leq \frac{1}{\eta^{2}} \operatorname{Var}\left[W_{n, j}\left(y_{2}\right)-W_{n, j}\left(y_{1}\right)\right] \leq \frac{1}{\eta^{2}} \mathrm{E}\left[\left(W_{n, j}\left(y_{2}\right)-W_{n, j}\left(y_{1}\right)\right)^{2}\right] \\
& \leq \frac{1}{\eta^{2}}\left(y_{2}-y_{1}\right)^{2},
\end{aligned}
$$

which follows from the fact that

$$
\begin{aligned}
\left|\cos \left(Y_{j}-y_{2}\right)-\cos \left(Y_{j}-y_{1}\right)\right| & =\left|2 \sin \left(\frac{2 Y_{j}-y_{2}-y_{1}}{2}\right) \sin \left(\frac{y_{1}-y_{2}}{2}\right)\right| \\
& \leq\left|y_{1}-y_{2}\right| .
\end{aligned}
$$

Here we used the inequality $|\sin x| \leq|x|$. Therefore $W_{n}$ converges weakly to a zero mean Gaussian process $W$ on $[0,2 \pi]$ with covariance function $\operatorname{Cov}\left(W\left(y_{1}\right), W\left(y_{2}\right)\right)=(1 / 2) \cos \left(y_{1}-y_{2}\right)$. By the continuous mapping theorem, see Theorem 5.1 in Billingsley (1968), the supremum of $\left|W_{n}\right|$ then converges weakly to the supremum of the absolute value of the limit process, which proves the lemma.

Lemma 2. With $V$ as in Theorem 1, we have

$$
\sup _{0 \leq x \leq 2 \pi}|W(x)| \stackrel{\mathcal{D}}{=} \frac{1}{2} \sqrt{2} V .
$$

Proof. Let $N_{1}$ and $N_{2}$ denote two independent standard normal random variables and let us define the process $\tilde{W}$ by $\tilde{W}=(\tilde{W}(x))_{x \in[0,2 \pi]}$, where

$$
\tilde{W}(x) \stackrel{\mathcal{D}}{=} \frac{1}{2} \sqrt{2}\left(N_{1} \cos x+N_{2} \sin x\right) .
$$

Since the covariance function $\operatorname{Cov}\left(W\left(x_{1}\right), W\left(x_{2}\right)\right)$ of the process $W$, given by $(1 / 2) \cos \left(x_{1}-x_{2}\right)$, equals $\operatorname{Cov}\left(\tilde{W}\left(x_{1}\right), \tilde{W}\left(x_{2}\right)\right)$ by

$$
\begin{array}{r}
\operatorname{Cov}\left(\frac{1}{2} \sqrt{2}\left(N_{1} \cos x_{1}+N_{2} \sin x_{1}\right), \frac{1}{2} \sqrt{2}\left(N_{1} \cos x_{2}+N_{2} \sin x_{2}\right)\right) \\
=\frac{1}{2}\left(\cos x_{1} \cos x_{2}+\sin x_{1} \sin x_{2}\right)=\frac{1}{2} \cos \left(x_{1}-x_{2}\right),
\end{array}
$$

it follows that $W \stackrel{\mathcal{D}}{=} \tilde{W}$.

Next write

$$
\begin{aligned}
\frac{1}{2} & \sqrt{2}\left(N_{1} \cos x+N_{2} \sin x\right) \\
& =\frac{1}{2} \sqrt{2} \sqrt{N_{1}^{2}+N_{2}^{2}}\left(\frac{N_{1}}{\sqrt{N_{1}^{2}+N_{2}^{2}}} \cos x+\frac{N_{2}}{\sqrt{N_{1}^{2}+N_{2}^{2}}} \sin x\right) \\
& =\frac{1}{2} \sqrt{2} \sqrt{N_{1}^{2}+N_{2}^{2}}(\cos \xi \cos x+\sin \xi \sin x) \\
& =\frac{1}{2} \sqrt{2} \sqrt{N_{1}^{2}+N_{2}^{2}} \cos (x-\xi),
\end{aligned}
$$


for a $\xi$ such that $\cos \xi=N_{1} / \sqrt{N_{1}^{2}+N_{2}^{2}}$ and $\sin \xi=N_{2} / \sqrt{N_{1}^{2}+N_{2}^{2}}$. The supremum of the absolute value of (77) is equal to $(1 / 2) \sqrt{2} \sqrt{N_{1}^{2}+N_{2}^{2}}=$ $(1 / 2) \sqrt{2} V$, where $V$ has a Rayleigh distribution. This entails (6) .

Lemma 3. Let $a_{n}=\sqrt{n} h^{-\lambda(1+\alpha)-\lambda_{0}+1}(\zeta(h))^{-1}$ denote the normalising sequence in Theorem 1. For $l=1,2,3$ we have

$$
a_{n}\left(R_{n}^{(l)}-\mathrm{E}\left[R_{n}^{(l)}\right]\right) \stackrel{\mathrm{P}}{\rightarrow} \mathbf{0}
$$

as $n \rightarrow \infty$ and $h \rightarrow 0$. Here $\mathbf{0}$ denotes the zero process on $[0,1]$.

Proof. To prove the lemma, we will apply Prohorov's theorem, and in particular Theorem 8.1 of Billingsley (1968). Firstly, notice that for a fixed $x$ the remainder terms $a_{n}\left(R_{n}^{(l)}(x)-\mathrm{E}\left[R_{n}^{(l)}(x)\right]\right)$ vanish in probability, which was proved in van Es and Uh (2005). This implies that the finite dimensional vectors of the processes $a_{n}\left(R_{n}^{(l)}-\mathrm{E}\left[R_{n}^{(l)}\right]\right)$ also converge in probability to null vectors. To establish tightness, we will again verify conditions of Theorem 12.3 of Billingsley (1968). Notice that when $x=0$, the sequence $a_{n}\left(R_{n}^{(l)}(0)-\mathrm{E}\left[R_{n}^{(l)}(0)\right]\right)$ is tight, since it converges to zero in probability. Furthermore, for an arbitrary positive $\eta$ we have

$$
\begin{aligned}
P\left(a_{n}\left|R_{n}^{(1)}\left(x_{2}\right)-\mathrm{E}\left[R_{n}^{(1)}\left(x_{2}\right)\right]-\left(R_{n}^{(1)}\left(x_{1}\right)-\mathrm{E}\left[R_{n}^{(1)}\left(x_{1}\right)\right]\right)\right| \geq \eta\right) \\
\leq \frac{a_{n}^{2}}{\eta^{2}} \operatorname{Var}\left[R_{n}^{(1)}\left(x_{2}\right)-R_{n}^{(1)}\left(x_{1}\right)\right] \\
=\frac{a_{n}^{2}}{\eta^{2}} \frac{1}{n} \operatorname{Var}\left[R_{n, 1}^{(1)}\left(x_{2}\right)-R_{n, 1}^{(1)}\left(x_{1}\right)\right] \\
\leq \frac{a_{n}^{2}}{\eta^{2}} \frac{1}{n} \mathrm{E}\left[\left(R_{n, 1}^{(1)}\left(x_{2}\right)-R_{n, 1}^{(1)}\left(x_{1}\right)\right)^{2}\right] \\
\leq \frac{a_{n}^{2}}{\eta^{2}} \frac{1}{C^{2}} \frac{1}{\pi^{2}} \frac{1}{n} h^{2\left(\lambda_{0}-1\right)}\left(x_{2}-x_{1}\right)^{2} \\
\quad \times K^{2}\left(\int_{\epsilon}^{1}\left(\frac{1-s}{h^{2}}\right) \phi_{w}(s) s^{-\lambda_{0}} \exp \left(s^{\lambda} /\left(\mu h^{\lambda}\right)\right) d s\right)^{2} \\
=K^{2} \frac{a_{n}^{2}}{\eta^{2}} \frac{1}{C^{2}} \frac{1}{\pi^{2}} \frac{1}{n} h^{2\left(\lambda_{0}-2\right)-2}\left(x_{2}-x_{1}\right)^{2} \\
\quad \times\left(\int_{\epsilon}^{1}(1-s) \phi_{w}(s) s^{-\lambda_{0}} \exp \left(s^{\lambda} /\left(\mu h^{\lambda}\right)\right) d s\right)^{2} \\
=O\left(\frac{1}{n} h^{2\left(\lambda_{0}-2\right)-2+2(2+\alpha) \lambda}(\zeta(h))^{2} a_{n}^{2}\right) \frac{1}{\eta^{2}}\left(x_{2}-x_{1}\right)^{2} \\
=O\left(h^{2(\lambda-2)}\right) \frac{1}{\eta^{2}}\left(x_{2}-x_{1}\right)^{2} \\
=O(1) \frac{1}{\eta^{2}}\left(x_{2}-x_{1}\right)^{2} .
\end{aligned}
$$


where $K$ is some constant. Here we used Lemma 5 of van Es and Uh (2005), which states that

$$
\begin{aligned}
\int_{\epsilon}^{1} s^{-\lambda_{0}}(1 & -s)^{\beta} \phi_{w}(s) \exp \left(s^{\lambda} /\left(\mu h^{\lambda}\right)\right) d s \\
& \sim A\left(\frac{\mu}{\lambda} h^{\lambda}\right)^{1+\alpha+\beta} \zeta(h) \Gamma(\alpha+\beta+1),
\end{aligned}
$$

and the fact that for $0 \leq s \leq 1$ and $0 \leq x_{1}<x_{2} \leq 1$ we have

$$
\begin{aligned}
\mid \cos & \left(s\left(\frac{X_{j}-x_{2}}{h}\right)\right)-\cos \left(\frac{X_{j}-x_{2}}{h}\right)-\cos \left(s\left(\frac{X_{j}-x_{1}}{h}\right)\right)+\cos \left(\left(\frac{X_{j}-x_{1}}{h}\right)\right) \mid \\
& =\left|\int_{x_{1}}^{x_{2}} \int_{s}^{1} \frac{\partial^{2}}{\partial u \partial v}\left\{\cos \left(v\left(\frac{X_{j}-u}{h}\right)\right)-\cos \left(\frac{X_{j}-u}{h}\right)\right\} d u d v\right| \\
& =\left|\int_{x_{1}}^{x_{2}} \int_{s}^{1}\left\{\frac{1}{h} \sin \left(v\left(\frac{X_{j}-u}{h}\right)\right)+v\left(\frac{X_{j}-u}{h^{2}}\right) \cos \left(\frac{X_{j}-u}{h}\right)\right\} d u d v\right| \\
& \leq \int_{x_{1}}^{x_{2}} \int_{s}^{1} \frac{1}{h^{2}}\left(\left|X_{j}\right|+1+h\right) d u d v \\
& \leq \frac{1}{h^{2}}\left(\left|X_{j}\right|+1+h\right)(1-s)\left|x_{1}-x_{2}\right| .
\end{aligned}
$$

Hence the process $a_{n}\left(R_{n}^{(1)}-\mathrm{E}\left[R_{n}^{(1)}\right]\right)$ is tight.

In order to prove tightness of the process $a_{n}\left(R_{n}^{(2)}-\mathrm{E}\left[R_{n}^{(2)}\right]\right)$, note that, as above, for positive $\eta$

$$
\begin{aligned}
P\left(a_{n} \mid R_{n}^{(2)}\right. & \left.\left(x_{2}\right)-\mathrm{E}\left[R_{n}^{(2)}\left(x_{2}\right)\right]-\left(R_{n}^{(2)}\left(x_{1}\right)-\mathrm{E}\left[R_{n}^{(2)}\left(x_{1}\right)\right]\right) \mid \geq \eta\right) \\
& \leq \frac{a_{n}^{2}}{\eta^{2}} \frac{1}{n} \mathrm{E}\left[\left(R_{n, 1}^{(2)}\left(x_{2}\right)-R_{n, 1}^{(2)}\left(x_{1}\right)\right)^{2}\right] \\
& \leq 4 \frac{a_{n}^{2}}{\eta^{2}} \frac{1}{4 \pi^{2} h^{2}} \frac{1}{n}\left(\int_{-\epsilon}^{\epsilon} \frac{s}{h} \phi_{w}(s) \frac{1}{\phi_{k}(s / h)} d s\right)^{2}\left(x_{2}-x_{1}\right)^{2} \\
& =4 \frac{a_{n}^{2}}{\eta^{2}} \frac{1}{4 \pi^{2} h^{4}} \frac{1}{n}\left(\int_{-\epsilon}^{\epsilon} s \phi_{w}(s) \frac{1}{\phi_{k}(s / h)} d s\right)^{2}\left(x_{2}-x_{1}\right)^{2} \\
& \leq 4 a_{n}^{2} \frac{1}{4 \pi^{2} h^{4}} \frac{1}{n}(2 \epsilon)^{2} \epsilon^{2}\left(\int_{-\epsilon}^{\epsilon} \frac{1}{\phi_{k}(s / h)} d s\right)^{2} \frac{1}{\eta^{2}}\left(x_{2}-x_{1}\right)^{2} \\
& \leq 4 a_{n}^{2} \frac{1}{\pi^{2} h^{4}} \frac{1}{n} \epsilon^{4}\left(\sup _{-\epsilon \leq s \leq \epsilon} \frac{1}{\phi_{k}(s / h)}\right)^{2} \frac{1}{\eta^{2}}\left(x_{2}-x_{1}\right)^{2} \\
& \leq 4 a_{n}^{2} \frac{2}{C^{2} \pi^{2}} \frac{1}{n}(\epsilon / h)^{4-2 \lambda_{0}} \exp \left(2(\epsilon / h)^{\lambda} / \mu\right) \frac{1}{\eta^{2}}\left(x_{2}-x_{1}\right)^{2} \\
& =o(1) \frac{1}{\eta^{2}}\left(x_{2}-x_{1}\right)^{2},
\end{aligned}
$$


where $K$ is some constant and where we used the fact that for $0 \leq s \leq 1$,

$$
\begin{aligned}
\left|\exp \left(i s\left(\frac{X_{j}-x_{2}}{h}\right)\right)-\exp \left(i s\left(\frac{X_{j}-x_{1}}{h}\right)\right)\right| \\
\quad \leq\left|\cos \left(s\left(\frac{X_{j}-x_{2}}{h}\right)\right)-\cos \left(s\left(\frac{X_{j}-x_{1}}{h}\right)\right)\right| \\
\quad+\left|\sin \left(s\left(\frac{X_{j}-x_{2}}{h}\right)\right)-\sin \left(s\left(\frac{X_{j}-x_{1}}{h}\right)\right)\right| \leq \frac{2 s}{h}\left|x_{1}-x_{2}\right|,
\end{aligned}
$$

which follows by converting the differences of sines and cosines into products and using the fact that $|\sin x| \leq|x|$. Consequently, the process $a_{n}\left(R_{n}^{(2)}-\right.$ $\left.\mathrm{E}\left[R_{n}^{(2)}\right]\right)$ is tight.

To prove tightness of the process $a_{n}\left(R_{n}^{(3)}-\mathrm{E}\left[R_{n}^{(3)}\right]\right)$, we first introduce the function $u$, given by

$$
u(y)=\frac{C|y|^{\lambda_{0}} \exp \left(-|y|^{\lambda} / \mu\right)}{\phi_{k}(y)}-1 .
$$

By Condition 1 this function is bounded on $\mathbb{R} \backslash(-\delta, \delta)$, where $\delta$ is an arbitrary positive number. Moreover, by (3) the function $x u(x)$ is also bounded and both functions vanish at plus and minus infinity. It follows that $(s / h) u(s / h)$ is bounded and tends to zero for all fixed $s$ with $|s| \geq \epsilon$ as $h \rightarrow 0$.

Using the function $u$, rewrite $R_{n, j}^{(3)}(x)$ as follows

$$
\begin{aligned}
R_{n, j}^{(3)}(x)= & \frac{1}{2 \pi h}\left(\int_{-1}^{-\epsilon}+\int_{\epsilon}^{1}\right) \exp \left(i s\left(\frac{X_{j}-x}{h}\right)\right) \phi_{w}(s) \\
& \times\left(\frac{1}{\phi_{k}(s / h)}-\frac{1}{C}\left(\frac{|s|}{h}\right)^{-\lambda_{0}} \exp \left(|s|^{\lambda} /\left(\mu h^{\lambda}\right)\right)\right) d s \\
=\frac{1}{2 \pi h}( & \left.\int_{-1}^{-\epsilon}+\int_{\epsilon}^{1}\right) \exp \left(i s\left(\frac{X_{j}-x}{h}\right)\right) \phi_{w}(s) \\
& \times \frac{1}{C}\left(\frac{|s|}{h}\right)^{-\lambda_{0}} \exp \left(|s|^{\lambda} /\left(\mu h^{\lambda}\right)\right) u(s / h) d s .
\end{aligned}
$$

Next note that, as above, for positive $\eta$ we have by (10) that

$$
\begin{aligned}
& P\left(a_{n}\left|R_{n}^{(3)}\left(x_{2}\right)-\mathrm{E}\left[R_{n}^{(3)}\left(x_{2}\right)\right]-\left(R_{n}^{(3)}\left(x_{1}\right)-\mathrm{E}\left[R_{n}^{(3)}\left(x_{1}\right)\right]\right)\right| \geq \eta\right) \\
& \leq \frac{a_{n}^{2}}{\eta^{2}} \frac{1}{n} \mathrm{E}\left[\left(R_{n, 1}^{(3)}\left(x_{2}\right)-R_{n, 1}^{(3)}\left(x_{1}\right)\right)^{2}\right] \\
& \leq \frac{a_{n}^{2}}{\eta^{2}} \frac{1}{4 \pi^{2} h^{2}} \frac{1}{n}\left(\left(\int_{-1}^{-\epsilon}+\int_{\epsilon}^{1}\right) \phi_{w}(s)\right. \\
&\left.\quad \times \frac{1}{C}\left(\frac{|s|}{h}\right)^{-\lambda_{0}} \exp \left(|s|^{\lambda} /\left(\mu h^{\lambda}\right)\right) \frac{s}{h} u\left(\frac{s}{h}\right) d s\right)^{2}\left(x_{2}-x_{1}\right)^{2} \\
&=o(1) \frac{1}{\eta^{2}}\left(x_{2}-x_{1}\right)^{2}
\end{aligned}
$$


and hence $a_{n}\left(R_{n}^{(3)}-\mathrm{E}\left[R_{n}^{(3)}\right]\right)$ is tight. By Prohorov's theorem each of the three processes now converges weakly to the zero process. Since the convergence in distribution to a constant entails convergence to the same constant in probability, this concludes the proof of the lemma.

Finally, we combine the obtained results to prove Theorem 1.

Proof of Theorem 1. The proof is immediate from Lemmas 13 just proved, the fact that by (9)

$$
\begin{gathered}
a_{n} \frac{1}{\pi C} h^{\lambda_{0}-1} \int_{\epsilon}^{1} \phi_{w}(s) s^{-\lambda_{0}} \exp \left(s^{\lambda} /\left(\mu h^{\lambda}\right)\right) d s \frac{1}{\sqrt{n}} \\
\sim \frac{A}{\pi C}\left(\frac{\mu}{\lambda}\right)^{1+\alpha} \Gamma(\alpha+1),
\end{gathered}
$$

and Theorems 4.1 and 5.1 of Billingsley (1968).

\section{References}

Bissantz, N., Dümbgen, L., Holzmann, H. and Munk, A., 2007. Nonparametric confidence bands in deconvolution density estimation. J. Roy. Statist. Soc. Ser. B 69, 483-506.

Bickel, P. J. and Rosenblatt, M., 1973. On some global measures of the deviations of density function estimates. Ann. Statist. 1, 1071-1095.

Billingsley, P., 1968. Convergence of Probability Measures. Wiley, New York.

Carroll, R. J. and Hall, P., 1988. Optimal rates of convergence for deconvolving a density. J. Amer. Stat. Assoc. 83, 1184-1186.

Carroll, R. J., Ruppert, D., Stefanski, L. A. and Crainiceanu, C. M., 2006. Measurement Error in Nonlinear Models: A Modern Perspective. Chapman \& Hall/CRC Press, Boca Raton, 2nd ed.

van Es, A. J. and Kok, A. R., 1998. Simple kernel estimators for certain nonparametric deconvolution problems. Statist. Probab. Lett. 39, 151160.

van Es, A. J. and and Uh, H.-W., 2004. Asymptotic normality of nonparametric kernel-type deconvolution density estimators: crossing the Cauchy boundary. J. Nonparametr. Stat. 16, 261-277.

van Es, A. J. and and Uh, H.-W., 2005. Asymptotic normality of kernel type deconvolution estimators. Scand. J. Statist. 32, 467-483.

Fan, J., 1991. Asymptotic normality for deconvolution kernel density estimators. Sankhyā Ser. A 53, 97-110. 
Fan, J., 1992. Deconvolution for supersmooth distributions. Canad. J. Statist. 20, 155-169.

Fan, J. and Liu, Y., 1997. A note on asymptotic normality for deconvolution kernel density estimators. Sankhyā Ser. A 59, 138-141.

Holzmann, H. and Boysen, L., 2006. Integrated square error asymptotics for supersmooth deconvolution. Scand. J. Statist. 33, 849-860.

Stefanski, L. A. and Carroll, R. J., 1990. Deconvoluting kernel density estimators. Statistics 2, 169-184.

Wand, M. P. and Jones, M. C., 1995. Kernel Smoothing. Chapman \& Hall, London. 